\title{
La ciencia abierta (open science) bajo el paraguas de Europa
}

\section{Open science under the umbrella of Europe}

\section{Ciência aberta sob o guarda-chuva da Europa}

\author{
Remedios Melero \\ Instituto de Agroquímica y Tecnología de Alimentos-CSIC, Valencia, España. \\ Email: rmelero@iata.csic.es.
}

Resumen: El significado de open access ha sido definido en numerosas ocasiones, tomando como referencia las declaraciones de Budapest, Bethesda y Berlín, también conocidas como las 3 BBB del open access. Sin embargo, no existe una única definición de open science, aunque sí existe una idea consensuada en cuanto a aceptar que "la ciencia abierta" implica una visión holística de todo el proceso de producción, comunicación y preservación de la producción científica. Por otro lado, la ciencia por definición es "abierta" de lo contario no hubiera habido avances científicos, por lo tanto, la colaboración entre grupos de trabajo, la difusión y acceso a los resultados y publicaciones es relevante para ese avance de la ciencia. En este artículo se pretende dar una visión general de lo que se está realizando en Europa para facilitar e incentivar el desarrollo de las infraestructuras y servicios que sirvan de sustento a la open science. Este apoyo estructural viene acompañado de políticas e iniciativas encaminadas a crear ese cambio cultural hacia una ciencia en la que compartir datos, publicar en acceso abierto, abrir el proceso de revisión, abrir las puertas a nuevas formas de evaluación académica basadas en nuevas métricas, y abrir las puertas a la ciudadanía formen parte de esa visión de ciencia responsable y compartida.

Palabras clave: Acceso abierto. Ciencia abierta. Políticas. Europa.

Abstract: The meaning of open access has been defined on numerous occasions, taking as reference the Budapest, Bethesda and Berlin Declarations, also known as the 3 BBB of open access. However, there is no single definition of open science, although there is a consensus on accepting that it implies a holistic view of the entire process of production, communication and preservation of scientific production. On the other hand, science is "open" by definition, otherwise there would have been no scientific advances; therefore, collaboration between working groups, dissemination and access to research results and publications is relevant to the advancement of science. This article aims to offer an overview of what is being done in Europe to facilitate and encourage the development of infrastructure and services supporting open science. This structural support is accompanied by policies and initiatives aimed at achieving a cultural change towards a science, where sharing data, publishing in open access, opening the review process, establishing forms of academic evaluation based on new metrics, and opening the doors to citizenship, are part of the vision of a responsible and shared science.

Keywords: Open access. Open science. Policys. Europe

Resumo: $\mathrm{O}$ significado de acesso aberto foi definido em várias ocasiões, tomando como referência as declarações de Budapeste, Bethesda e Berlim, também conhecidas como o acesso aberto de 3 BBB. No entanto, não existe uma definição única de ciência aberta, embora haja um consenso em aceitar que a "ciência aberta" implica uma visão holística de todo o processo de produção, comunicação e preservação da produção científica. Por outro lado, a ciência, por definição, é "aberta", caso contrário não teria havido avanços científicos, portanto, a colaboração entre grupos de trabalho, a disseminação e o acesso a resultados e publicações é relevante para esse avanço na ciência. Este artigo tem como objetivo dar uma visão geral do que está sendo feito na Europa para facilitar e incentivar o desenvolvimento de infraestrutura e serviços que sirvam de apoio à ciência aberta. Esse apoio 
estrutural é acompanhado por políticas e iniciativas destinadas a criar essa mudança cultural rumo a uma ciência na qual compartilhar dados, publicar em acesso aberto, abrir o processo de revisão, abrir as portas para novas formas de avaliação acadêmica baseadas em novas métricas e abrir as portas para a cidadania fazem parte dessa visão de ciência responsável e compartilhada.

Palavras-chave: Acesso aberto. Ciência aberta. Políticas. Europa

\section{Introducción al concepto de Open Science en el contexto europeo}

En 2014 la Comisión Europea hizo una encuesta online abierta a todas las partes integrantes del paisaje de la investigación en Europa. Los objetivos de la encuesta fueron: evaluar el grado de conocimiento entre las partes interesadas en el cambio del modus operandi hacia una ciencia abierta; evaluar la percepción de las oportunidades y desafíos de la open science; identificar las posibles implicaciones en las políticas científicas, y analizar las acciones para fortalecer la competitividad del sistema europeo de ciencia e investigación. La encuesta se hizo en términos de "ciencia 2.0" y entre sus cuestiones estaba la de elegir un término que se ajustara al concepto de investigación abierta de acuerdo a la definición anterior (European Commision, 2015). De los resultados, se obtuvo que el término preferido para definir la ciencia en abierto fue "open science" en lugar de "science 2.0". Por lo tanto, a partir de este momento la Comisión utiliza en su documentación e informes el término open science para referirse a un nuevo enfoque del proceso científico basado en el trabajo cooperativo y en nuevas formas de difundir el conocimiento mediante el uso de tecnologías digitales y nuevas herramientas de colaboración.

Entre los resultados de la consulta anterior se detectaron cuáles eran las posibles acciones impulsoras de la open science y cuales las barreras para su implementación. En las Figuras 1 y 2 se muestran algunos de estos resultados, entre los que destacan las posibilidades que brindan las tecnologías digitales como motor de la ciencia abierta, y cómo los obstáculos se ciernen en las dudas sobre la garantía de calidad y la falta de reconocimiento. Estos resultados fueron obtenidos en el 2014, después de cuatro años, algunas de las cuestiones siguen vigentes, pero la percepción de que la open science está más asentada entre la comunidad científica, ha mejorado y las barreras para su implementación han disminuido al mejorar la infraestructura y servicios de apoyo a los investigadores. 
Figura 1. Acciones que impulsan la open science

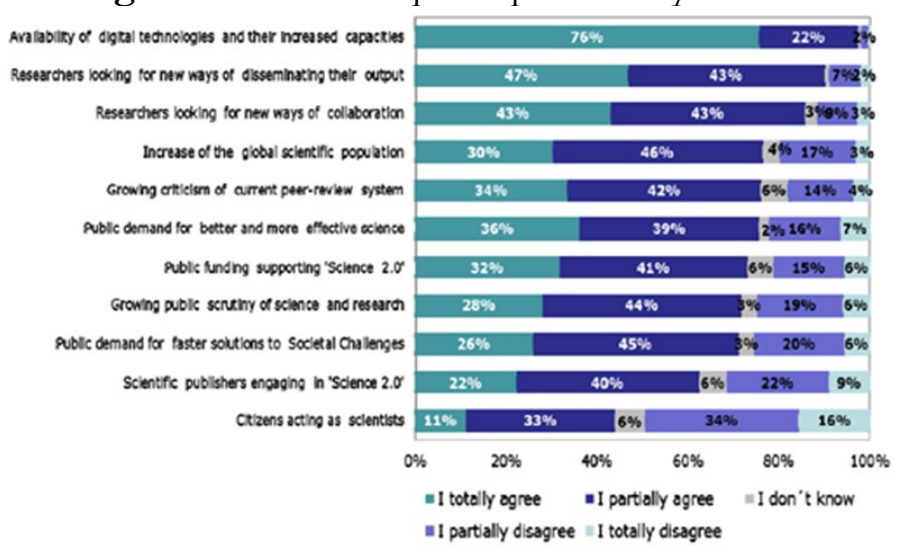

Fuente: European Commission (2015).

Figura 2. Barreras que impiden el desarrollo de la open science

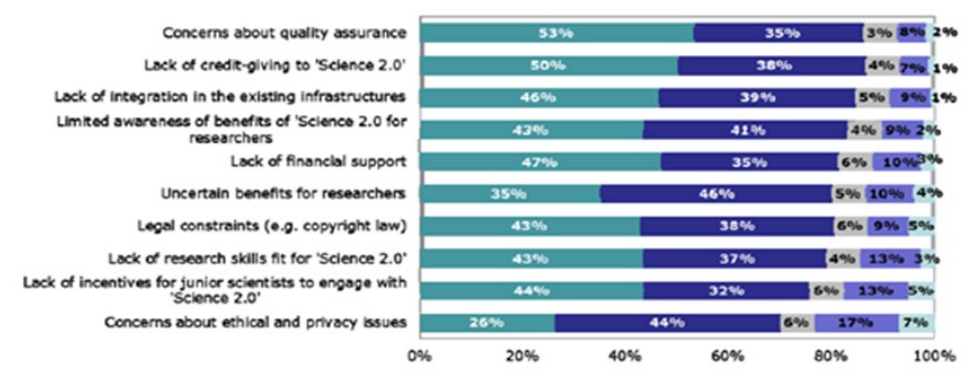

Fuente: European Commission (2015).

En 2015 la OCDE, definía la ciencia abierta como "los esfuerzos para hacer que los resultados de la investigación financiada con fondos públicos sean más accesibles para la comunidad científica, el sector empresarial o para la sociedad en general" (OCDE, 2015). En síntesis, podemos decir que la ciencia abierta insta a abrir todo el ciclo de la investigación para favorecer la transparencia, la reproducibilidad, la colaboración y la reutilización de los resultados. La open science abarca diferentes "opens" (Figura 3) entre ellos el open access y open data en los que me centraré en este artículo. 
IRIS)

La ciencia abierta (open science) bajo el paraguas de Europa

Remedios Melero

Figura 3. Envolvente de la ciencia abierta conformada por los diferentes "open"

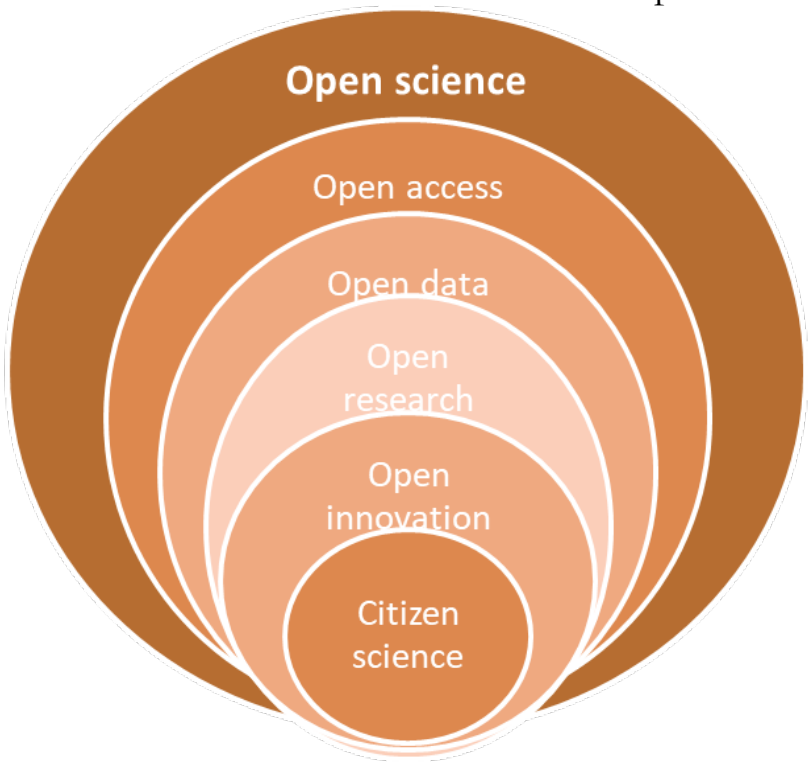

Fuente: Elaboracion propria

En resumen, el concepto de open science supone un cambio sistémico en la forma en que la ciencia y la investigación se ha llevado a cabo durante los últimos cincuenta años: pasar de las prácticas habituales de publicación de resultados de investigación en publicaciones científicas, a compartir y utilizar todo el conocimiento disponible en las distintas fases de la investigación tal y como se plasmó en el documento Open science, open innovation, open to the world (EUROPEAN COMMISSION, 2016a). Teniendo en cuenta este nuevo paradigma de entender la ejecución y comunicación de la ciencia, este artículo pretende dar una visión general de lo que se está realizando en Europa para facilitar e incentivar el desarrollo de las infraestructuras y servicios que sirvan de sustento a la open science.

\section{Políticas europeas que promueven la ciencia abierta}

En general, la mayoría de países europeos tienen sus propias políticas de acceso abierto, a nivel local, nacional, de institución académica o de agencias financiadoras (SparcEurope, 2018). Algunas de ellas han evolucionado a una política en favor de la ciencia abierta a nivel nacional por la cual se comprometen a implementar medidas encaminadas a favorecer la open science. Recientemente, por ejemplo, el ministro francés para la ciencia anunció su plan nacional para la ciencia abierta el 4 de julio de 2018 en un encuentro de LIBER, que contempla tres pilares para su implementación: el acceso abierto a las publicaciones, los datos de investigación en abierto y la integración de la ciencia abierta en la actividad cotidiana del investigador" (FRÉDÉRIQUE VIDAL, 2018). En el caso español, el nuevo ministro Pedro Luque de 
Ciencia Investigación y Universidades, anunció la intención de trabajar en el diseño de la Estrategia Nacional de Ciencia en Abierto "coherente con la política europea de Open science y seguir trabajando en la implementación de la ley de la ciencia en su capitulo de acceso abierto a las publicaciones, los repositorios y su interoperabilidad".

Holanda, durante su periodo de presidencia de la Unión Europea en 2016, impulsó de forma muy activa iniciativas y políticas encaminadas a la open science. Así se puso de manifiesto en la reunión de Ámsterdam con representantes políticos y académicos, en la que se acordaron una serie de acciones para abordar la open science como fuente de generación de conocimiento, innovación y riqueza, para lo cual se establecieron dos metas importantes a alcanzar en 2020:

1. Acceso abierto total a todas las publicaciones científicas;

2. Un enfoque nuevo que contribuya a la reutilización óptima de los datos de investigación

También se fijó una estrategia para alcanzar esos objetivos basada en establecer nuevos sistemas de reconocimiento y evaluación de la actividad científica y una alineación de políticas e intercambio de buenas prácticas (MINISTRY OF EDUCATION, CULTURE AND SCIENCE, 2016).

Por otro lado, en el borrador para la agenda digital europea se definieron 5 acciones para la open science (European Commission, 2016b):

- Fomentar la ciencia aberta;

- Eliminar las barreras a la open science;

- Desarrollar infraestructuras de apoyo a una investigación abierta;

- Acceso abierto a los resultados de investigación;

- Integrar la ciencia abierta en la sociedad.

Estas cinco acciones serán los pilares en los que se basarán las futuras políticas que aboguen por hacer que las publicaciones científicas estén en abierto y se facilite que los datos de investigación cumplan con los principios FAIR (findable, accesible, interoperable and reusable). Por supuesto, siempre que su reutilización se haga de una forma responsable, y estén tan 
abiertos como sea posible y tan cerrados como sea necesario ("as open as possible, as closed as necessary").

En resumen, Europa aboga por un futuro en el que la reutilización de los resultados de la investigación favorezca la innovación y el desarrollo. Para cumplir con estos objetivos la Comisión Europea ha creado una serie de instrumentos basados en el asesoramiento, la infraestructura y la creación de directrices o estándares para hacer que los objetos digitales del ecosistema de la open science sean interoperables. Para el asesoramiento ha creado un grupo de expertos, la Open science Policy Platform (EOSPP) que se encarga de elaborar informes y recomendaciones relacionadas con la implementación de la open science en Europa. Esta comisión se nombró en septiembre de 2016 y hasta ahora han emitido distintos documentos sobre la European Open Science Cloud (EOSC), la publicación open access, las nuevas métricas, posibles perfiles de profesionales para la gestión de datos, e incentivos para los investigadores que adopten prácticas de ciencia abierta en su trabajo.

Respecto a la infraestructura, la Comisión financia proyectos ESFRI (European Strategy Forum on Research Infrastructures) encaminados a crear una masa crítica de infraestructuras dedicadas a la investigación. Estos proyectos están relacionados con la European Open Science Cloud, cuyo objetivo es crear un entorno de apoyo a la open science: "The European Open science Cloud is a supporting environment for Open science and not an 'open cloud' for science".

Respecto a los estándares, y herramientas de análisis de datos, la Comisión Europea ha financiado distintos proyectos con servicios de apoyo a los investigadores teniendo en cuenta también la disciplina (Science Europe, 2018), como por ejemplo ELIXIR (https://www.elixireurope.org/) en bioinformática, CLARIN (https://www.clarin.eu/) en ciencias sociales y humanidades o EUDAT (https://www.eudat.eu/) de apoyo multidisciplinar.

\section{European Open Science Cloud (EOSC)}

El anuncio de la creación de la EOSC se hizo en el año 2016. La EOSC es un proyecto europeo cuyo objetivo es la creación de un entorno virtual con servicios para el almacenamiento, gestión, análisis y reutilización de datos de investigación. Está concebida como una nube de servicios de apoyo a los investigadores (Figura 4) basada en la federación de infraestructuras existentes en los países miembros. Para su desarrollo se apoya en otros proyectos y se espera que las directrices para su gobernanza y estructura estén acabadas en el año 2022 (Figura 5). 
Figura 4. Infografía sobre la European Open Science Cloud

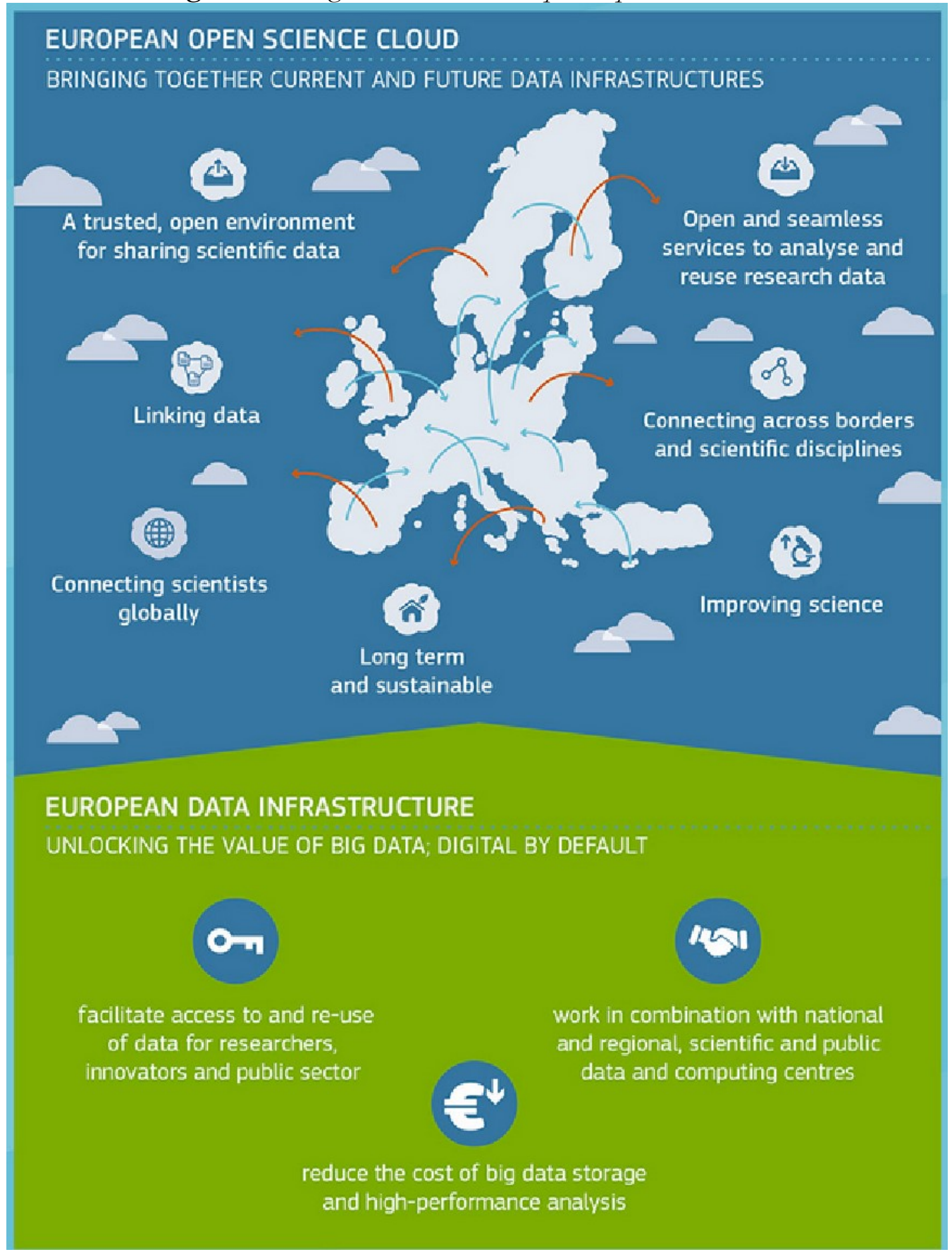

Fuente: EOSC (2016) 
Figura 5. Cronología para el desarrollo de la EOSC.

\begin{tabular}{|c|c|}
\hline & $2016>2017>2018>2019>2020>2021>2022$ \\
\hline \multicolumn{2}{|l|}{ EOSCpilot } \\
\hline \multicolumn{2}{|l|}{ EOSC-hub } \\
\hline \multicolumn{2}{|l|}{ OpenAIRE-Advance } \\
\hline \multicolumn{2}{|l|}{ FREYA } \\
\hline \multicolumn{2}{|l|}{ elnfraCentral } \\
\hline \multicolumn{2}{|l|}{$\begin{array}{l}\text { Commercial services } \\
\text { access }\end{array}$} \\
\hline \multicolumn{2}{|l|}{$\begin{array}{l}\text { Connecting ESFRI } \\
\text { infrastructures }\end{array}$} \\
\hline \multirow{2}{*}{\multicolumn{2}{|c|}{$\begin{array}{l}\text { Support to the EOSC } \\
\text { Governance CSA }\end{array}$}} \\
\hline & \\
\hline \multirow{2}{*}{\multicolumn{2}{|c|}{$\begin{array}{l}\text { Prototyping new } \\
\text { innovative services }\end{array}$}} \\
\hline & \\
\hline \multirow{2}{*}{\multicolumn{2}{|c|}{$\begin{array}{l}\text { Support to the EOSC } \\
\text { Governance RIA }\end{array}$}} \\
\hline & \\
\hline Enhancing the EOSC & \\
\hline portal & \\
\hline
\end{tabular}

Fuente: EOSC (2016)

El EOSC pilot project supone la primera fase del desarrollo de la EOSC y sus objetivos son proponer las líneas de funcionamiento o gobernanza de la EOSC, desarrollar proyectos pilotos que integren servicios e infraestructuras que muestren la interoperabilidad en diferentes áreas científicas, e implicar a comunidades científicas a adquirir la confianza y las capacidades necesarias para la adopción de nuevos hábitos conducentes a una ciencia abierta. 


\section{Políticas de Acceso Abierto de la Comisión Europea a las publicaciones y a los datos} de investigación

La Comisión Europea en el Séptimo Programa Marco (2007-2013) impulsó el acceso abierto a las publicaciones mediante un plan piloto que afectó a un $20 \%$ del total de la financiación y que abarcaba sietes áreas: 1. Energía 2. Medio ambiente (incluido el cambio climático). 3. Salud. 4. Tecnologías de la información y comunicación (sistemas cognitivos, interacción, robótica). 5. Infraestructuras de investigación (e-infraestructuras) 6. Ciencia en la sociedad 7. Ciencias socioeconómicas y humanidades (EUROPEAN COMMISION, 2008). De acuerdo al plan piloto, los trabajos publicados, resultantes del proyecto financiado, deberían depositarse en un repositorio institucional o temático lo antes posible y con un máximo permitido de embargo de 12 meses para ciencias sociales y humanidades y de 6 meses para el resto de disciplinas. La base de la convocatoria permitía que en el caso de publicar en una revista con APCs (author processing charges), los gastos derivados de esa publicación pudieran justificarse como costes directos del proyecto. Incluso hubo una convocatoria posterior, para costear aquellos trabajos pendientes de publicar (Post-grant Open Access Pilot) en revistas que cobraban por publicar.

Para la monitorización del piloto, el proyecto OpenAire puso en marcha una herramienta para hacer el seguimiento del cumplimiento del mandato de open Access, en función de los artículos en abierto respecto a los publicados. La información se recolecta de los repositorios institucionales, a partir de los metadatos que contienen el código de proyecto o información similar siguiendo unas directrices (OpenAIRE, 2015).

Las estadísticas de OpenAIRE revelan que todavía no se ha alcanzado el 100\% del open access a las publicaciones sujetas al Piloto (Figura 6).

En el siguiente programa marco, Horizon 2020 (2014-2020), la política open access de la Comisión se extendió a todas las disciplinas, deja de ser un piloto, y se mantienen los periodos de embargo permitidos igual que en el anterior Programa Marco (EUROPEAN COMMISSION, 2016). 
Figura 6. Publicaciones en abierto sujetas al proyecto piloto de acceso abierto del Séptimo Programa Marco

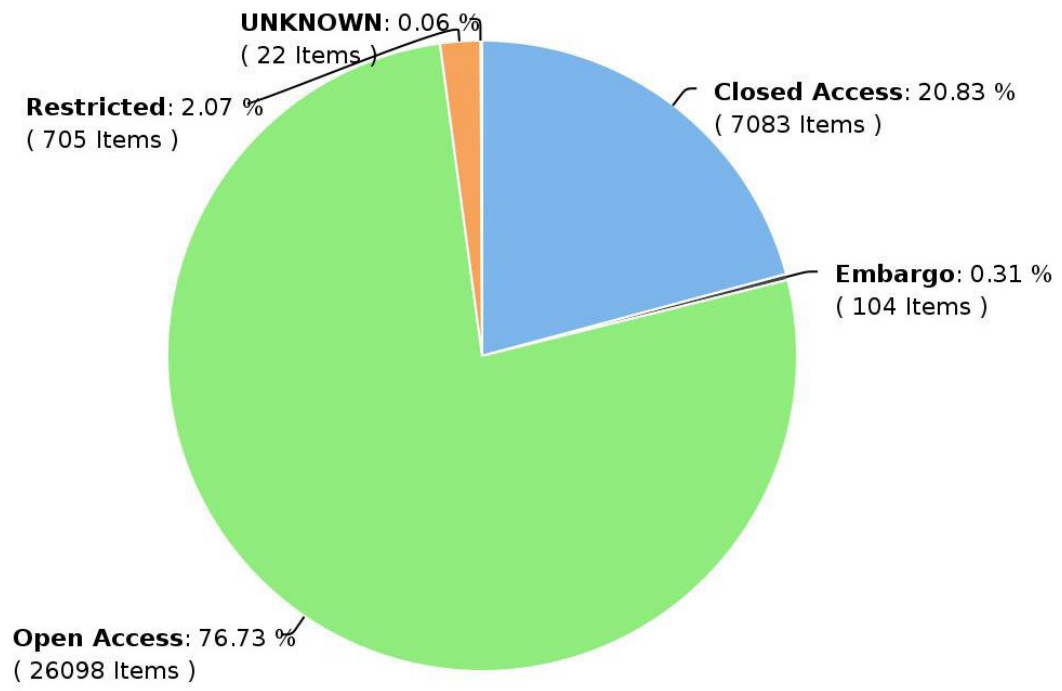

Fuente: OpenAIRE, https://www.openaire.eu/fp7-stats).

Respecto al cumplimiento del acceso abierto a las publicaciones las estadísticas al día de hoy, y teniendo en cuenta que todavía no ha concluido el H2020 (Figura 7) muestran que el porcentaje de acceso abierto es ligeramente mayor, y lo que ha disminuido considerablemente son las ratios de acceso restringido y cerrado, aunque hay cerca del 20\% de acceso desconocido. 
Figura 7. Publicaciones en abierto sujetas al mandato de acceso abierto del programa H2020

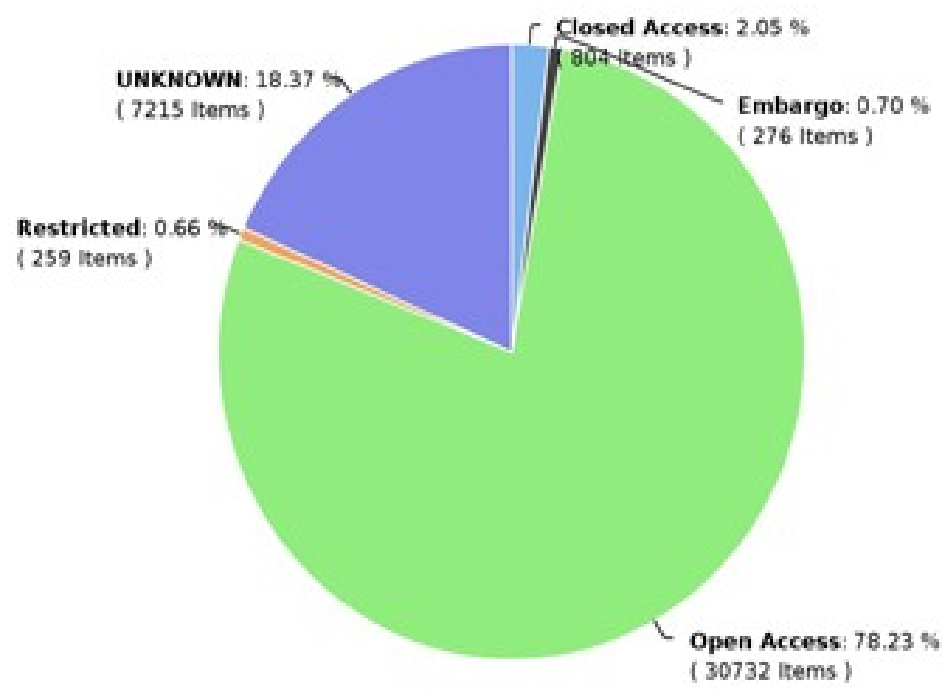

Fuente: OpenAIRE, https://www.openaire.eu/h2020-stats.

En el Horizon 2020 se inicia un plan piloto con los datos de investigación. Por un lado, los proyectos de investigación deben presentar un plan de gestión de datos a los 6 meses de inicio del proyecto y cada vez que se haga una revisión del mismo. Por otro, aquellos datos subyacentes a los trabajos publicados u otros que se especifiquen en el plan de gestión de datos, deben depositarse en un repositorio institucional o temático de manera que sean localizables, accesibles, interoperables y reutilizables (datos FAIR). El plan piloto afectó hasta el 1 de enero de 2017 a determinadas disciplinas y, a partir de 2017, se ven implicadas todas las áreas para los nuevos proyectos concedidos a partir de esa fecha (EUROPEAN COMMISSION, 2017).

Los datos pueden quedar exentos de ser compartidos en diferentes escenarios, entre ellos:

- Si los resultados esperados son susceptibles de ser explotados comercial o industrialmente;

- Si existen razones de confidencialidad o de seguridad;

- Si la exposición de los datos es incompatible con alguna normativa referente a la protección de datos personales; 
- Si la exposición de los datos pone en riesgo el desarrollo del objetivo principal del proyecto;

- Si el proyecto no prevé la generación o colección de datos;

- Si existe alguna otra razón legítima para no participar en el Piloto.

La exención puede expresarse en la solicitud del proyecto o bien durante su ejecución, y deben exponerse las razones en el plan de gestión de datos.

\section{Proyectos europeos relacionados con la Open science}

Europa ha financiado proyectos tanto de infraestructura como de servicios dirigidos al acceso y preservación de la producción científica. Su número es alto y su descripción algo ardua, como ejemplo, en la Figura 8 se muestran algunos (EUROPEAN UNION, 2016)

Figura 8. Algunos proyectos financiados por la Comisión Europea relacionados con la open science.

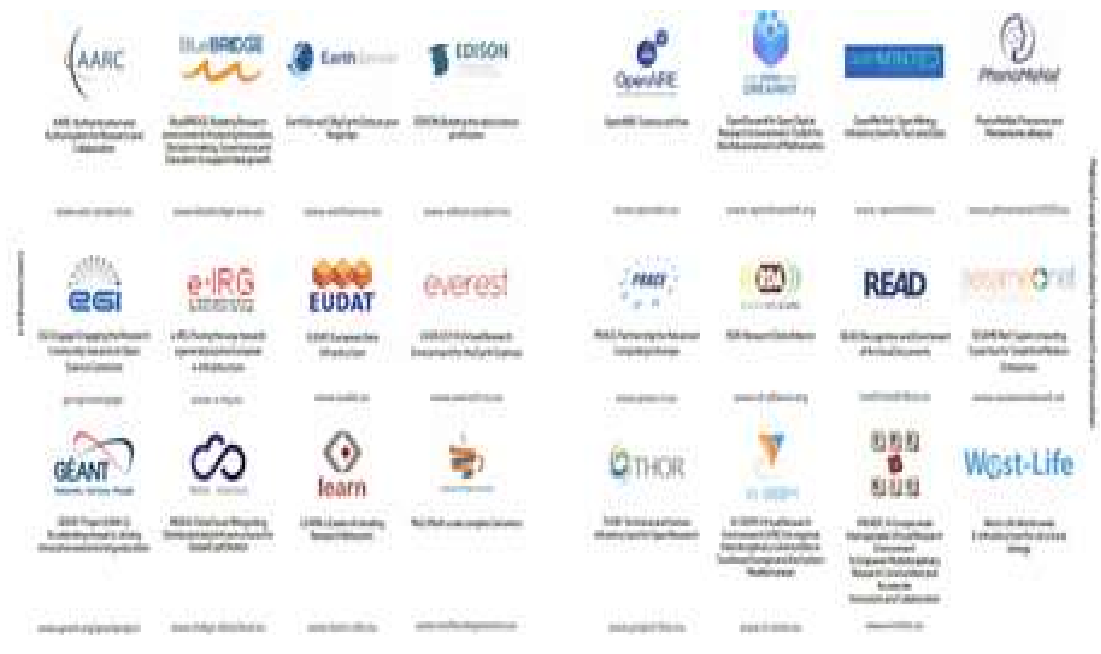

De algunos proyectos que todavía están vigentes he escogido los siguientes por su interés y aplicación directa a algún componente de la open science. Todos ofrecen numerosas herramientas y materiales para formación (online y presencial): FOSTER+, OpenUp, OpenMinTED, OpenAIRE, GO FAIR y FREYA.

a) FOSTER+ (https://www.fosteropenscience.eu/) 
El Proyecto FOSTER+ tiene por objetivo general dar a difundir las políticas europeas de ciencia abierta, y proporcionar a través de la formación y la creación de recursos y materiales, una capacitación específica dirigida a los investigadores.

Esta fase del proyecto está orientada a las ciencias naturales, ciencias sociales y humanidades. Entre los recursos generados del proyecto, cabe destacar el manual Open science Training Handbook cuyo objetivo es apoyar a los capacitadores en contenido y en la forma de llevar a cabo cursos de formación en temas de Open science. El portal de FOSTER dispone también de una sección de recursos referentes a eventos y bibliografía actualizada sobre temas incluidos en la taxonomía propuesta por sus socios (ver Figura 9)

Figura 9. Taxonomía de open science creada por el proyecto FOSTER.

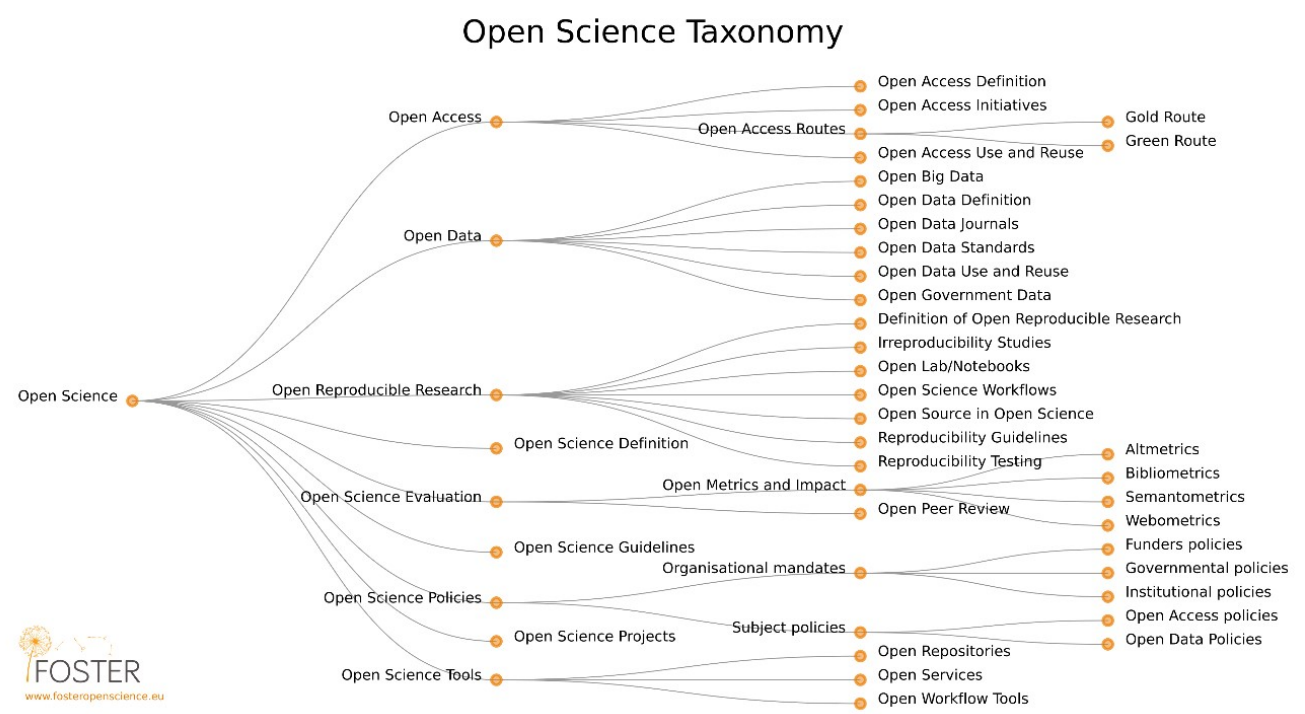

Otros productos creados recientemente son los cursos online para seguirlos de forma autónoma. La versión final de los cursos saldrá en otoño tras analizar los comentarios recibidos en el periodo de prueba (https://www.fosteropenscience.eu/toolkit).

\section{b) OpenUp (http://openup-h2020.eu/)}

Este proyecto se centra en el análisis de los nuevos métodos de evaluación (peer review) de la calidad científica, nuevas métricas del impacto científico y nuevas formas de diseminación de los resultados de investigación. 
IRIS)

La ciencia abierta (open science) bajo el paraguas de Europa

Remedios Melero

Ha creado una plataforma, OpenUp Hub, que recolecta recursos, facilita guías, casos prácticos, vídeos y otros materiales, centrados en los temas de peer review, evaluación y comunicación de la producción científica.

c) OpenMinTeD (https://services.openminted.eu/home)

Los socios de OpenMinTeD han creado una e-Infraestructura abierta, orientada a servicios para la minería de textos y datos (Text and Data Mining) de contenido científico y académico. Los investigadores pueden crear, descubrir, compartir y reutilizar de forma colaborativa el conocimiento de una amplia gama de fuentes textuales científicas de forma sencilla. La plataforma de servicios (https://services.openminted.eu/home) permite hacer minería de datos en la nube con una selección de aplicaciones, tomando fuentes de referencia publicaciones open access.

\section{d) OpenAIRE (https://www.openaire.eu ).}

El proyecto OpenAIRE ha pasado por varias fases desde el año 2009: OpenAIRE, OpenAIRE+, OpenAIRE2020. No es un proyecto propiamente dicho sino un portal de servicios a investigadores, instituciones académicas y agencias de financiación. Está formado por representantes de 50 nodos europeos. Establece estándares para la interoperabilidad entre repositorios, hace un seguimiento del depósito en repositorios de publicaciones de proyectos financiados por la Comisión Europea, facilita recursos y capacitación en temas de open access y open data. Actualmente están en fase de desarrollo marcha y OpenAIRE Advance y OpenAire Connect. El primero servirá de apoyo, como hasta ahora, en el cumplimiento de las políticas de la Comisión Europea de open access y open data, y el segundo facilitara la infraestructura para crear una red de linked data entre datos, publicaciones, proyectos e investigadores.

e) GO FAIR ( https://www.go-fair.org/).

GO FAIR ofrece un ecosistema abierto e inclusivo para individuos, instituciones y organizaciones comprometidas con la definición y creación de materiales y herramientas orientados a los datos de investigación.

Es una iniciativa de apoyo a la EOSC basada en el trabajo colaborativo de comunidades científicas agrupadas en forma de redes temáticas. Actualmente está en fase de desarrollo y cuenta con la opción de poder formar parte de esas redes de acuerdo a unos 
determinados criterios (https://www.go-fair.org/implementation-networks/starting-newimplementation-network/process/).

\section{f) FREYA ( https://www.project-freya.eu/).}

Es un proyecto continuación del anterior THOR (https://project-thor.eu/) y tiene como objetivo central construir una infraestructura interoperable basada en identificadores permanentes, integrante de la ciencia abierta. Sus servicios se construirán sobre la infraestructura existente de Crossref, DataCite, ORCID e identifiers.org, con esto se pretende mejorar la localización, la recuperación y el acceso a la producción científica.

\section{Consideraciones Finales}

En un documento publicado en abril de 2018 por la Comisión Europea, relativo al acceso a la información científica y a su preservación, se hacen unas recomendaciones con el fin de incentivar y promover la ciencia abierta, que se pueden resumir brevemente en los siguientes puntos (EUROPEAN COMMISSION, 2018):

- Acceso abierto a las publicaciones científicas;

- Gestión de los datos de investigación, incluyendo el acceso abierto;

- Preservación y reutilización de la información científica;

- Infraestructuras para la ciencia abierta;

- Capacidades y competencias;

- Incentivos y recompensas;

- Diálogo multilateral sobre la ciencia abierta a nivel nacional, europeo e internacional;

- Coordinación estructurada de los Estados miembros a nivel de la UE y seguimiento de la presente recomendación.

Casi simultáneamente a este documento, la Liga Europea de Universidades (LERU) publicó también un informe en el que se hacían unas recomendaciones globales dirigidas a las instituciones (LERU, 2018):

- Nombrar a un gestor senior que lidere las iniciativas Open science entorno a estos 8 pilares; 
- Desarrollo de un programa para promover el cambio cultural;

- Desarrollo de programas que promuevan las oportunidades que brinda la open science;

- Trazar una estrategia de comunicación que permita familiarizar a la comunidad científica con las prácticas open science.

También se realizaron recomendaciones específicas orientadas a:

- Facilitar infraestructura;

- Apoyo (recursos humanos);

- Reconocimiento y nuevas métricas;

- Incentivos;

- Políticas institucionales/gubernamentales acordes a prácticas open science;

- Integridad y transparencia;

- Seguimiento/monitorización de las políticas;

- Formación/capacitación;

- Participación de la comunidad científica (responsabilidad social, nuevas prácticas);

- Participación de la ciudadanía;

- Cambio cultural.

En resumen, la Unión Europea no solo es un motor económico; la ciencia y la innovación juegan papeles importantes en su visión de una sociedad del futuro más responsable y respetuosa con nuestro entorno. Por ello, la ciencia abierta es una apuesta importante por su propio significado, abierta, colaborativa y compartida.

\section{Referencias}

EUROPEAN COMMISSION. Open Access Pilot in FP7. 2008. Disponível em:

$<$ http:/ /ec.europa.eu/research/swafs/index.cfm?pg=policy\&lib=pilot>. Acesso em: 30 jul. 2018.

EUROPEAN COMMISSION. Validation of the results of the public consultation on Science 2.0: Science in Transition. 2015. Disponível em: $<$ http://www.eesc.europa.eu/resources/docs/validation-of-the-results-of-the-public-consultation-onscience-20.pdf. >. Acesso em: 30 jul. 2018. 
EUROPEAN COMMISSION.Open science, open innovation open to the world. 2016a Disponível em: < http://ec.europa.eu/newsroom/dae/document.cfm?doc id=16022. doi:10.2777/061652. >. Acesso em: 30 jul. 2018.

EUROPEAN COMMISSION. Draft European Open science Agenda. 2016b Disponible: https://ec.europa.eu/research/openscience/pdf/draft_european_open_science_agenda.pdf.

EUROPEAN COMMISSION. Guidelines to the rules on open access to scientific publications and open access to research data in H2020. 2017. Disponível em:

<http://ec.europa.eu/research/participants/data/ref/h2020/grants_manual/hi/oa_pilot/h2020-hioa-pilot-guide_en.pdf $>$. Acesso em: 30 jul. 2018.

EUROPEAN COMMISSION. Commission recommendation (EU) 2018/790 of 25 April 2018 on access to and preservation of scientific information. 2018. Disponível em: $<$ https://eurlex.europa.eu/legal-content/EN/TXT/?uri=CELEX:32018H0790>. Acesso em: 30 jul. 2018.

EUROPEAN UNION. Making Europe the best place for research and innovation. 2016. Disponível em:

$<$ http://ec.europa.eu/newsroom/dae/document.cfm?action=display\&doc_id=16543.) >. Acesso em: 30 jul. 2018.

MINISTRY OF EDUCATION, CULTURE AND SCIENCE. Amsterdam Call for Action on Open science. 2016. Disponível em:

$<$ https://www.government.nl/binaries/government/documents/reports/2016/04/04/amsterdamcall-for-action-on-open-science/amsterdam-call-for-action-on-open-science.pdf $>$. Acesso em: 30 jul. 2018.

OCDE . Making Open science a Reality. OECD Science, Technology and Industry Policy Papers, n. 25, OECD Publishing, Paris, 2015. Disponível em: < https://doi.org/10.1787/5jrs2f963zs1-en. >. Acesso em: 30 jul. 2018.

OpenAIRE. OpenAIRE Guidelines for Literature Repositories. 2015. Disponível em: <https://guidelines.openaire.eu/en/latest/literature/index.html>. Acesso em: 30 jul. 2018.

SCIENCE EUROPE. Guidance Document 2018, Presenting a Framework for Discipline-specific.

Reserch Data Mangement, 2018. Disponível em: < content/uploads/2018/01/SE Guidance Document RDMPs.pdf>. Acesso em: 30 jul. 2018.

SPARC EUROPE. Open Data and Open science Policies in Europe. 2018. Disponível em: <https://sparceurope.org/download/2285/>. Acesso em: 30 jul. 2018.

VIDAL, Frederique. Plan national pour la science ouverte: discours de Frédérique Vidal. 2018. Disponível em: <http://www.enseignementsup-recherche.gouv.fr/cid132531/plan-national-pour-lascience-ouverte-discours-de-frederique-vidal.html). >. Acesso em: 30 jul. 2018.

OPEN SCIENCE Policy Platform. Disponível em:

$<$ https://ec.europa.eu/research/openscience/index.cfm?pg=open-science-policy-platform>. Acesso em: 30 jul. 2018.

ESFRI European Strategy Forum on Research Infrastructures Disponível em: <http://www.esfri.eu/>. Acesso em: 30 jul. 2018. 
EUROPEAN OPEN SCIENCE CLOU. Disponível em: < https://eoscpilot.eu/>. Acesso em: 30 jul. 2018.

EOSC Pilot. Disponível em: <https://www.eoscpilot.eu/about-eoscpilot>. Acesso em: 30 jul. 2018.

Data de submissão: $31-07-2018$

Data de aceitação: 21-09-2018 